\title{
PESQUISA E EXTENSÃO NA CONSERVAÇÃO DE MANGUEZAIS DA ILHA DE SANTA CATARINA
}

Originais recebidos em: 18/01/2011

Aceito para publicação em: 11/01/2012

Bianca Pinto Vieira

Universidade Federal de Santa Catarina biancabioufsc@gmail.com

Elaine Mitie Nakamura

Universidade Federal de Santa Catarina elainemitie@gmail.com

Dayse Dias

Universidade Federal de Santa Catarina daysediasgrad@gmail.com

Natalia Hanazaki

Universidade Federal de Santa Catarina natalia@ccb.ufsc.br

\section{Resumo}

Este trabalho relata as intervenções socioambientais realizadas a partir dos resultados de uma pesquisa sobre o encalhe de resíduos no manguezal, estudo este desenvolvido por uma equipe de acadêmicos do Curso de Ciências Biológicas da Universidade Federal de Santa Catarina (UFSC), de outubro de 2008 a junho de 2010. Foram realizadas atividades de pesquisa e educação ambiental em prol da conservação de manguezais, destinação adequada de resíduos sólidos urbanos e redução na produção destes. A pesquisa envolveu doze meses de coleta de dados em campo sobre o encalhe de resíduos sólidos e intervenções socioambientais, as quais se deram através de diversas palestras, oficinas, cursos e materiais didáticos destinados a diferentes grupos sociais. Nesse processo, os acadêmicos difundiram ideias e levantaram questões para reflexão em um diversificado público de Florianópolis.

Palavras-chave: Educação ambiental. Mangue. Resíduos sólidos. Pontal do Jurerê. Florianópolis.

\section{RESEARCH AND EXTENSION FOR MANGROVE CONSERVATION}

\section{Abastract}

This article aims to present the social and environmental interventions performed from the results of a research about solid waste stranding in mangrove. This work was developed by an academic team from Biological Sciences' Course at Federal University of Santa Catarina (UFSC) from October 2008 to June 2010. The group had made researches and environmental education activities for mangrove conservation, solid waste reduction and proper discard of it. The research involved a year of field data collection about solid waste stranding. The environmental education was made possible through activities like occasional lectures, workshops, courses and didactic materials production. With these actions the team spread ideas and raised issues to think about in a varied community of Florianopolis city.

Keywords: Environmental education. Mangrove. Solid waste. Pontal do Jurerê. Florianópolis. 


\section{INTRODUÇÃO}

Manguezais são ecossistemas singulares e possuem intensas relações ecológicas com diversas espécies, desde algas microscópicas a grandes mamíferos (SCHAEFFER-NOVELLI; CINTRÓN, 1986; SPALDING et al., 1997). A alta produtividade pesqueira desse meio possibilita a extração controlada de peixes, ostras, camarões, caranguejos e berbigões (HAMILTON; SNEDAKER, 1984). Além disso, proporciona o desenvolvimento de atividades turísticas e educação ambiental, tais como canoagem, trekking e outras (HAMILTON; SNEDAKER, 1984).

Indiretamente, entre as muitas funções dos manguezais, estes auxiliam: (i) na retenção de sedimentos, impedindo o assoreamento de canais de navegação; (ii) na redução do impacto da maré sobre áreas habitadas; e (iii) na absorção de boa parte da matéria orgânica (esgoto) e metais pesados descartados por atividades humanas (SCHAEFFER-NOVELLI; CINTRÓN, 1986). Porém, mesmo com esses aspectos que fundamentam a conservação dos manguezais (SPALDING et al., 1997; RODRIGUES et al., 2008), ainda é complexo trabalhar essa questão junto a grupos sociais, em especial aqueles que não convivem diretamente com o ecossistema em questão.

Ações de educação ambiental têm mostrado bons resultados, ressaltando a consciência ecológica coletiva através de aspectos legislativos, informativos e formativos, com a sensibilização do indivíduo para que este perceba a intensa relação existente entre o ser humano e o ambiente (SCHEINER, 1984). Contudo, ações de educação ambiental são praticadas de forma pontual, geralmente em comunidades próximas aos manguezais, buscando a forma mais simples de resgatar a percepção dos participantes sobre o ambiente e trabalhar as associações desse ecossistema com o cotidiano social de cada um (JAGTAP et al., 1993; O’NEAL, 1995; PARANAGUÁ et al., 2001; SILVA; SOUSA, 2004; BARCELLOS et al., 2005; SEDOVIM et al., 2005; PEREIRA et al., 2006; FARRAPEIRA et al., 2007; CARNEIRO et al., 2008; MELO et al., 2008; RODRIGUES et al., 2008; SEGALLA, 2008; PINHEIRO et al., 2010).

Neste estudo, partiu-se de um diagnóstico ambiental com base em Vieira et al. (2011a, 2011b) e da busca de referências sobre os temas envolvidos no processo de cognição 
ambiental em relação aos manguezais. Assim, buscou-se sensibilizar o público-alvo sobre as necessidades dos manguezais da Ilha de Santa Catarina como meio de contribuir com a percepção desse ecossistema como elemento biológico, social, cultural, político e econômico essencial às comunidades. Este artigo objetiva relatar as atividades desenvolvidas no Projeto Manguezal por uma equipe de acadêmicos do Curso de Ciências Biológicas da UFSC em Florianópolis - SC.

\section{MATERIAL E MÉTODOS}

De outubro de 2008 a outubro de 2009 , foram realizadas coletas mensais de resíduos sólidos urbanos no manguezal de Ratones. Foram considerados resíduos sólidos urbanos os objetos descartados formados por matéria em estado sólido, resultantes de atividades humanas domiciliares e que, por suas particularidades, exigem soluções técnicas adequadas para destinação final (BRASIL, 2010). Os dados obtidos publicados por Vieira et al. (2011a, 2011b) forneceram subsídios para desenvolver intervenções socioambientais com diversos setores da sociedade local.

A estratégia de atuação deste trabalho envolveu ações com diferentes grupos, a fim de atingir o maior público possível. Inicialmente, visando aos usuários da internet, montou-se um sítio eletrônico (www.projetomanguezal.ufsc.br) com informações sobre o projeto, sobre os manguezais do Brasil e do mundo, resíduos sólidos, educação ambiental e conservação. Outros materiais utilizados em palestras, cursos e oficinas para a comunidade acadêmica da UFSC foram fôlderes e pôsteres informativos, bem como apresentações digitais sobre temas tais como a conservação dos manguezais brasileiros e os impactos dos resíduos sólidos em ecossistemas naturais. Esses materiais também foram trabalhados junto a moradores das regiões de manguezais da Ilha de Santa Catarina, estudantes de ensino fundamental, professores do ensino público e frequentadores de praias de Florianópolis.

Como forma de atingir efetivamente o público-alvo, foi adotada uma abordagem baseada na união de informação e experiência, com atividades teóricas e práticas, visando estimular a reflexão pessoal de cada um, com propostas instigantes, curiosas e artísticas. Mesmo com o término das coletas, em outubro de 2009, as atividades de extensão continuaram até junho de 2010 com palestras, cursos, saídas de campo e oficinas para escolas públicas e outras entidades da Ilha de Santa Catarina - a exemplo da Prefeitura Municipal de Florianópolis, da Escola do Mar e do Grupo de Escoteiros dos Ingleses. 


\section{RESULTADOS E ANÁLISE}

A partir das coletas no manguezal (Figura 1), verificou-se que a maioria dos resíduos é composta por plásticos (63\%, de 602 itens coletados), e há uma tendência de estes se acumularem nas áreas mais próximas de residências e em bordas de capim-praturá (Spartina alterniflora Loisel) do manguezal (VIEIRA et al., 2011a). Nas coletas mensais foram retirados resíduos sólidos urbanos, como portas, fogões, plásticos, redes de nylon, isopor, cordas, borrachas e vidros (Figura 2), auxiliando na limpeza do manguezal. A partir desses resultados sobre a avaliação de encalhe de resíduos sólidos urbanos, foram executadas ações de extensão relacionadas ao tema.

A primeira das realizações de contribuição para com a comunidade e demais frequentadores das praias locais foi o evento de limpeza do Pontal de Jurerê, localizado em Florianópolis - SC, em março de 2009, juntamente com a Estação Ecológica de Carijós (ESEC Carijós). O evento durou um dia e teve a participação de 40 acadêmicos do curso de Ciências Biológicas. Foram recolhidas três toneladas de resíduos dos ecossistemas de praia, restinga e manguezal do Pontal do Jurerê.

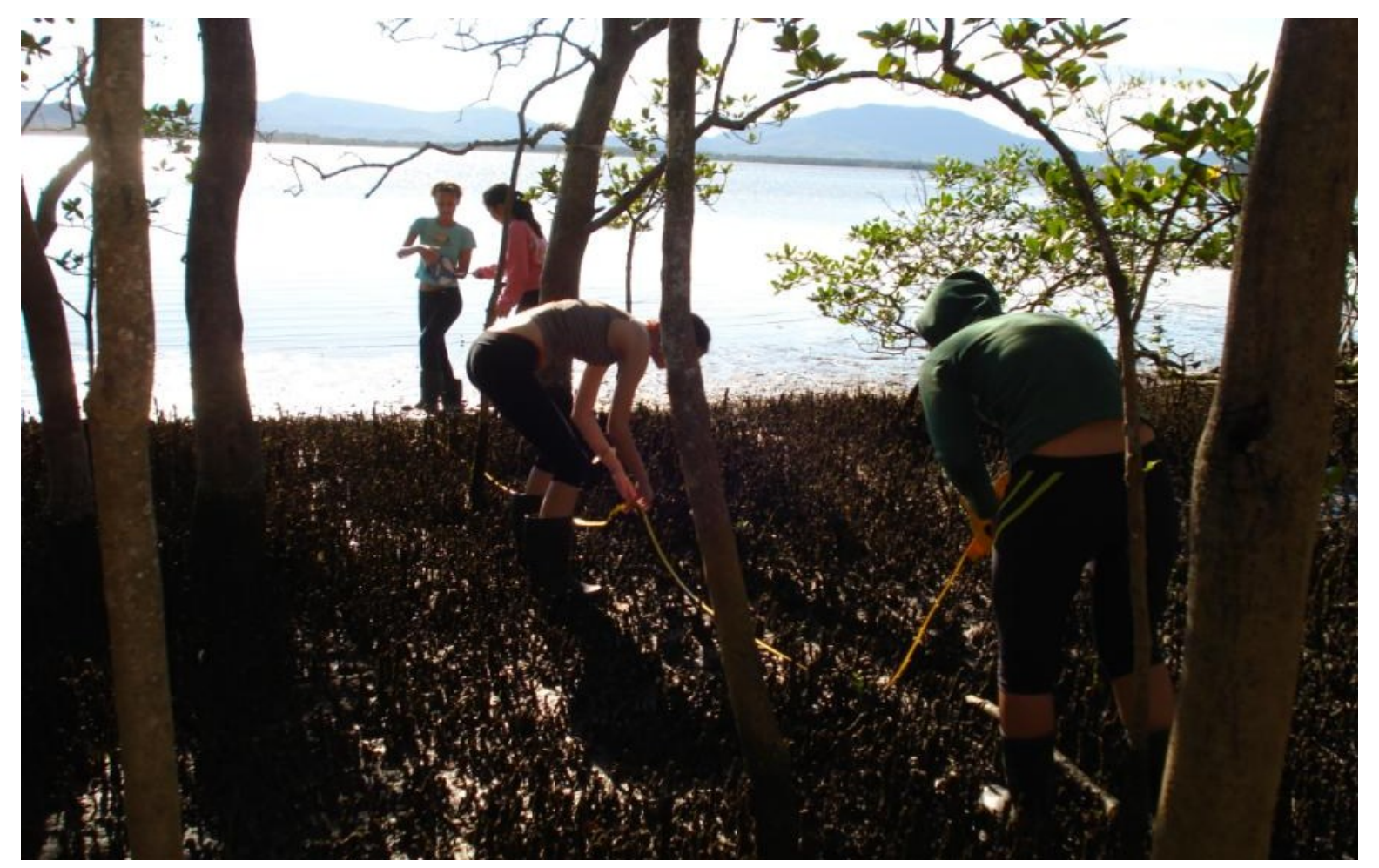

Figura 1: Coleta de resíduos sólidos no manguezal do Pontal do Jurerê. 


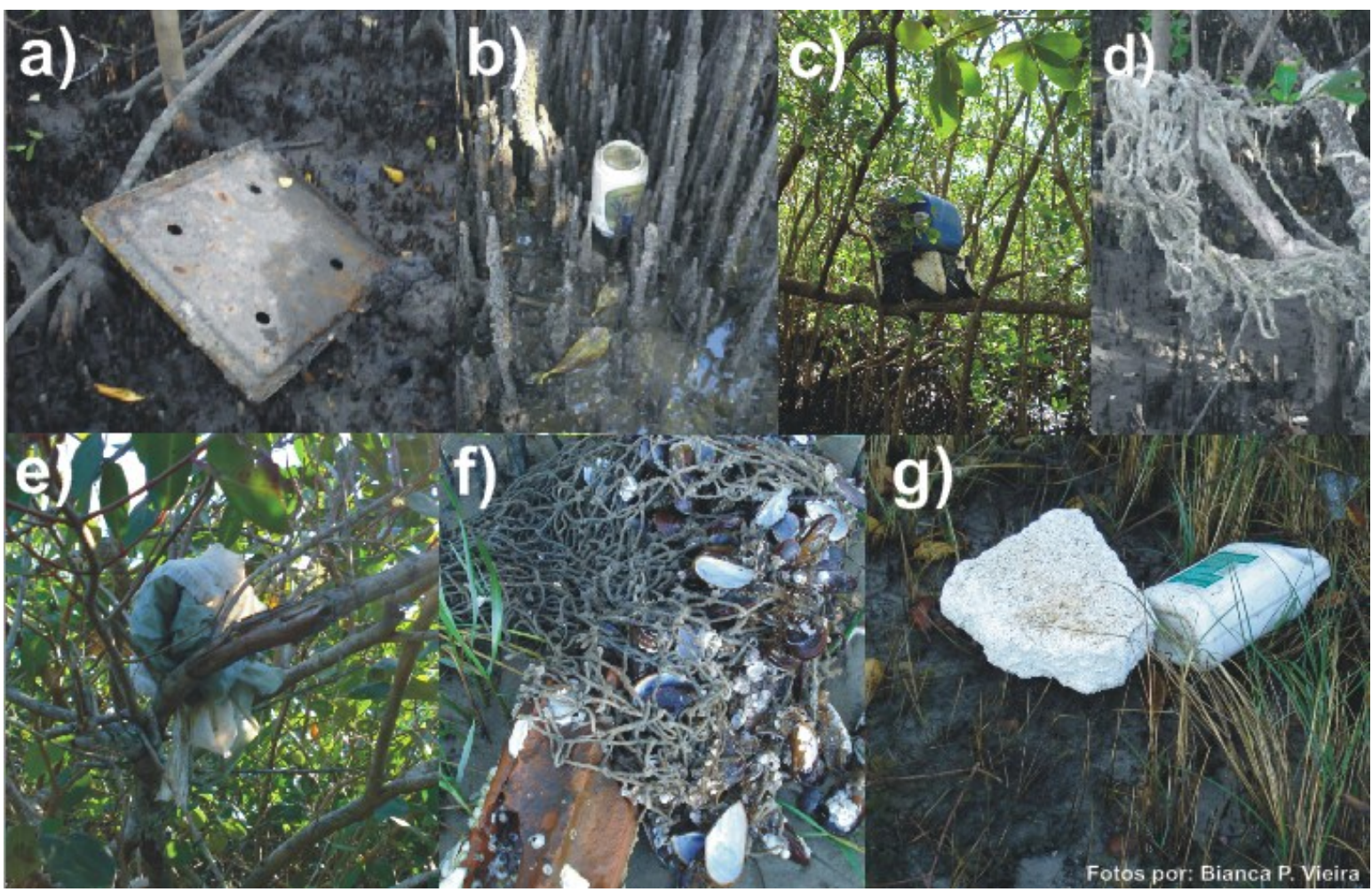

Figura 2: Diversos resíduos sólidos encontrados no manguezal do Pontal de Jurerê, Florianópolis - SC. a) Tampa de fogão encontrada em mangue-vermelho (Rhizophora mangle L.). b) Lata de alumínio em pneumatóforo. c) Boia de isopor e plástico no alto de mangue-branco (Laguncularia racemosa (L.) C. F. Gaertn). d) Rede presa em mangue-preto. e) Plástico no alto de mangue-preto (Avicennia schaueriana Stapf \& Leechm. ex Moldenke). f) Rede e tijolo com mariscos e cracas. g) Isopor e garrafa plástica de produto tóxico em capim-praturá (Spartina alterniflora Loisel).

Por convite do Instituto Carijós Pró-Conservação da Natureza, foram ministradas conferências em junho de 2009, com o tema "Manguezal: ensinar e aprender", em cursos de capacitação para professores da rede municipal de ensino público de Florianópolis. Palestras também foram oferecidas aos acadêmicos do curso de Ciências Biológicas da UFSC, na disciplina Introdução à Ecologia (ECZ7012), em junho de 2009 e abril de 2010; ao Grupo de Discussões em Biologia da Conservação (GDBIC), em agosto de 2009; e ao Grupo Tecendo Educação Ambiental e Estudos Culturais, em setembro de 2009. Intervenções na disciplina Fotografia para Cinema, do curso superior de tecnologia em Fotografia da Universidade do Vale do Itajaí (UNIVALI), em abril de 2010, resultaram na produção de um vídeo educativo sobre os impactos do consumismo nos manguezais, disponível no sítio eletrônico Youtube (www.youtube.com/watch?v=v9xm23Qo7uA).

Na $8^{\text {a }}$ Semana de Ensino, Pesquisa e Extensão (SEPEX) da UFSC e no projeto de extensão Bio na Rua, o projeto realizou a distribuição de fôlderes, apresentação de trabalho 
(pôster) e slideshow em estande. Também foram atrações a exposição de um aquário vivo apresentando o ambiente de manguezal em menor escala e a realização do minicurso "Manguezal e seus diversos aspectos", oferecido para a comunidade em geral.

Foram realizadas oficinas para alunos da $5^{\mathrm{a}}$ série $\left(6^{\circ}\right.$ ano $)$ do ensino fundamental da Escola Básica Municipal Osmar Cunha. Através de uma abordagem instigante e lúdica, os estudantes da escola tiveram uma recepção muito calorosa das atividades artísticas desenvolvidas. O material cultural incluiu textos sobre folclore, música, poesia, quadrinhos e imagens sobre o ecossistema de manguezal. Os alunos se dividiram em cinco grupos e receberam materiais impressos sobre os manguezais no formato de quadrinhos, música, folclore, poesia e imagens. A partir do material recebido, as crianças deveriam se inspirar e expressar o que pensavam e sentiam sobre o manguezal. Como resultado, os alunos realizaram apresentações teatrais, musicais, desenhos e explicações sobre as possíveis histórias contidas nos materiais.

Por fim, em maio e junho de 2010, palestras, atividades com flanelógrafo - painel interativo com elementos referentes ao ecossistema de manguezal - e saídas de estudos atingiram todos os membros do Grupo de Escoteiros dos Ingleses (Figura 3). 

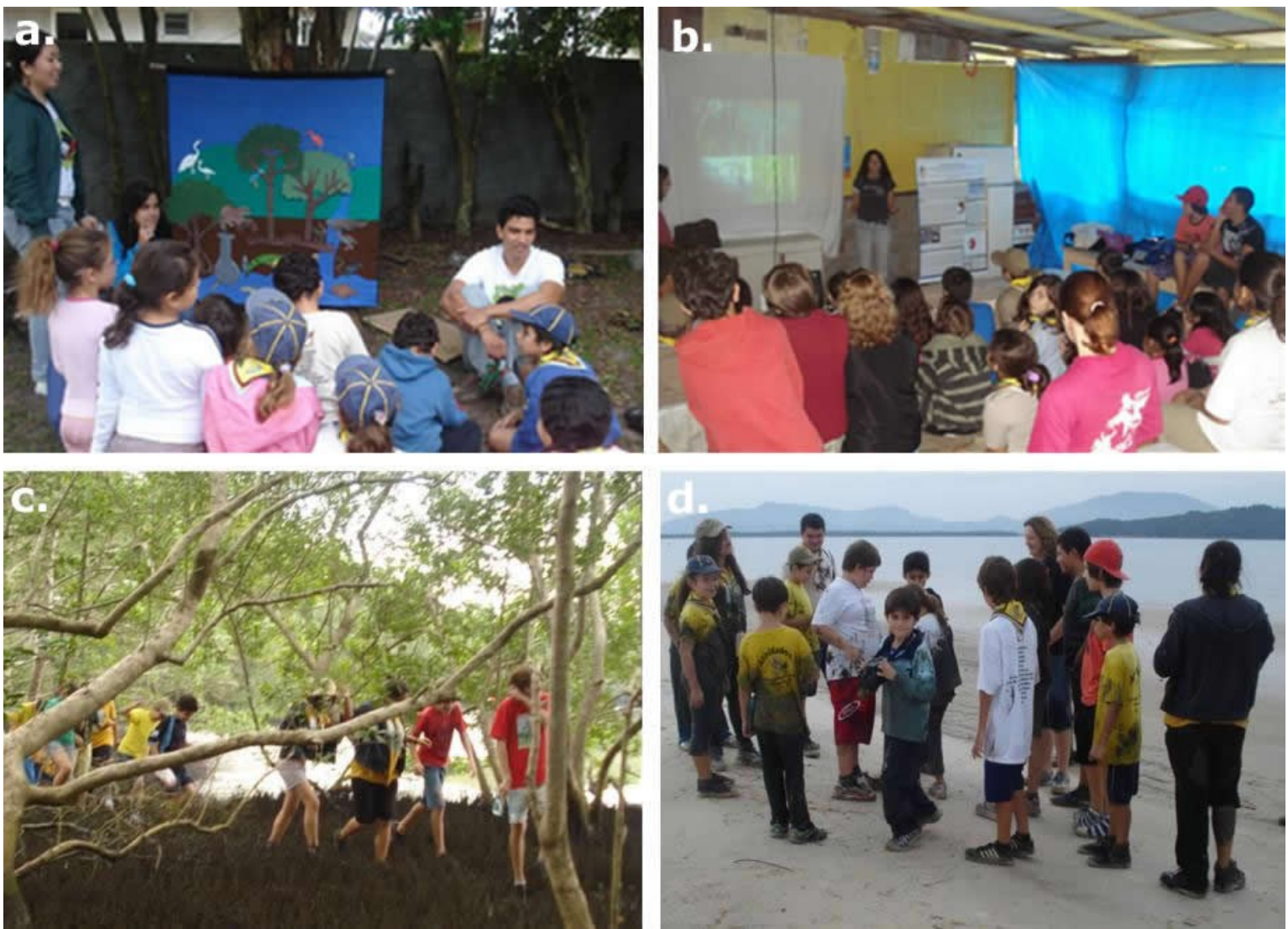

Figura 3: Atividades realizadas com o Grupo de Escoteiros dos Ingleses. a) Atividade no flanelógrafo com o grupo de lobinhos. b) Palestra para o grupo de escoteiros. c) Saída ao manguezal de Ratones com todos os membros do grupo. d) Parada na praia do Pontal ao final da saída de campo para conversar sobre a importância do que foi ensinado.

A difusão das atividades chamou a atenção da imprensa universitária, e algumas matérias foram realizadas junto à TV UFSC, ao jornal e ao site da Agência de Comunicação da UFSC.

\section{CONSIDERAÇÕES FINAIS}

Os manguezais são fundamentais na dinâmica costeira. Porém, ainda é difícil sensibilizar as pessoas em relação à importância desse ecossistema e de sua influência no seu dia a dia. Há muito a ser desenvolvido quanto à conscientização da comunidade para ações efetivas. Poucas são as iniciativas para sensibilização da sociedade em relação aos muitos problemas enfrentados por este ambiente, principalmente quanto ao acúmulo de resíduos.

Assim como diversos outros ecossistemas, os manguezais podem ser considerados apenas recursos a serem utilizados até sua extinção. Entretanto, também podem ser vistos 
como um ambiente passível de ser usado moderadamente, para que possa ser conservando para as futuras gerações. A conservação da saúde e do equilíbrio ambiental é extremamente importante para a promoção da saúde humana. Ações de extensão universitária possuem caráter fundamental na conscientização de diferentes públicos quanto à importância dos ecossistemas naturais. É preciso que as comunidades, em especial aquelas que vivem nos entornos desses meios, sintam-se engajadas em protegê-los para usufruir não só de um benefício próprio, mas também de um benefício coletivo. Para isso, é essencial a participação popular em projetos integrados de conservação que levem em consideração aspectos socioeconômicos das comunidades. Ressalta-se que nessa participação a população não deve limitar-se apenas a ouvir resoluções de cientistas e políticos, mas sim efetivar um termo no qual se sinta realmente responsável pelo bem ambiental em parceria com o órgão competente da região. As ações educativas e políticas exigem abordagens verdadeiramente interdisciplinares, a exemplo do que já é proposto em lei para as unidades de conservação de uso sustentável, como as Reservas Extrativistas.

Para que integração e interação sejam efetivas, é necessário auxiliar as comunidades a elucidarem seus próprios conhecimentos sobre o ambiente. Nesse contexto, os projetos de pesquisa e extensão universitária devem ser facilitadores para que a população se conscientize sobre o que já conhece dos manguezais, bem como devem ajudar na composição de estratégias e encurtar distâncias entre meio acadêmico, gestores públicos e comunidade. Desse modo, ações de extensão ou intervenções socioambientais integradas ao desenvolvimento de projetos de pesquisa facilitam a incorporação de contextos importantes para conservação por parte das comunidades. Tais ações também podem multiplicar as experiências com outros públicos, constituindo redes de socialização de conhecimentos.

É importante mencionar que, com suporte nos dados científicos obtidos pelo projeto e em outros dados sobre rejeitos urbanos, membros do projeto participaram da audiência pública sobre o estabelecimento de uma Estação de Tratamento de Esgoto (ETE) na Barra do Sambaqui, em fevereiro de 2009. Durante a audiência, a equipe argumentou a favor do deslocamento da ETE para uma área de menor impacto, pois a implantação no local prejudicaria a população e o ecossistema. A sugestão foi atendida e o sistema de coleta de esgoto da região foi aperfeiçoado. 
Espera-se que iniciativas como as do Projeto Manguezal sejam cada vez mais comuns e que os órgãos de fomento aumentem a incidência de apoio a linhas de pesquisa nesse âmbito, a fim de que se cumpram os objetivos de conservação e desenvolvimento deste importante ecossistema no Brasil. Considerando os impactos das ações antrópicas nos manguezais, são imprescindíveis, dentro da comunidade universitária, mais pesquisas voltadas à proteção desse sistema, aliadas a ações de extensão em curto, médio e longo prazo no entorno dos manguezais.

\section{REFERÊNCIAS}

BARCELlOS, P. A. O.; JUNIOR, S. M. A.; MUSIS, C. R.; BASTOS, H. F. B. N. 2005. As representações sociais dos professores e alunos da Escola Municipal Karla Patrícia, Recife, Pernambuco, sobre o manguezal. Ciência \& Educação, vol. 11, nº. 02, pp.213-222.

BERKES, F. 2005. Sistemas sociais, sistemas ecológicos e direitos de apropriação de recursos naturais. In: VIEIRA, P. F.; BERKES, F.; SEIXAS, C. S. Gestão Integrada e Participativa de Recursos Naturais: Conceitos, Métodos e Experiências. $1^{\mathrm{a}}$ ed., pp.47-72, Editora Secco, Florianópolis, SC, Brasil.

BRASIL. Lei $\mathrm{n}^{\circ}$. 12. 305, de 2 de agosto de 2010. Institui a Política Nacional de Resíduos Sólidos; altera a Lei n⿳0 9.605, de 12 de fevereiro de 1998; e dá outras providências. Disponível em <http://www.planalto.gov.br/ccivil_03/_ato2007-2010/2010/lei/112305.htm>. Acesso em: 18 out. 2010.

CARNEIRO, M. A. B.; FARRAPEIRA, C. M. R.; SILVA, K. M. E. 2008. O manguezal na visão etnoecológica dos pescadores artesanais do Canal de Santa Cruz, Itapissuma, Pernambuco, Brasil. Biotemas, vol. 21, nº. 04, pp.147-155.

FARRAPEIRA, C. M. R.; SILVA, K. M. E.; LIMA, A. O. 2007. Percepção e concepção do manguezal vinculados ao ensino da Biologia em uma escola de Recife - PE. Educação Ambiental em Ação, vol. 19, pp.1-9.

HAMILTON, L. S.; SNEDAKER, S. C. 1984. Handbook for mangrove area management. UNEP, Honolulu, USA, 123pp.

JAGTAP, T. G.; CHAVAN, V. S.; UNTAWALE, A. G. 1993. Mangrove Ecosystems of India: A Need for protection. Ambio, vol. 22, no. 4, pp.252-254.

MELO, A. V. O. M.; FARRAPEIRA, C. M. R.; PINTO, S. L. 2008. Estratégias de educação ambiental sobre o manguezal junto a uma comunidade estudantil de Olinda - PE. Revista Eletrônica do Mestrado em Educação Ambiental, vol. 21, pp.356-376. 
O’NEAL, L. H. 1995. Using Wetlands to Teach Ecology \& Environmental Awareness in General Biology. The American Biology Teacher, vol. 57, nº. 3, pp.135-139.

PARANAGUÁ, M. N.; ALMEIDA, V. L. S.; MELO-JÚNIOR, M.; ALVES, M. S.; BARROS, H. M. 2011. Educação ambiental como instrumento de gestão comunitária de ecossistemas manguezais do canal de Santa Cruz (PE, Brasil). Tropical Oceanography, vol. $39, \mathrm{n}^{\mathrm{o}} .1, \mathrm{pp} .14-21$.

PINHEIRO, M. A. A.; SANTOS, C. M. H.; WUNDERLICH, A. C.; MILÃO-SILVA, F.; PERES-COSTA, W. C. 2010. Educação ambiental sobre manguezais na baixada santista: uma experiência da UNESP/CLP. Revista Ciência em Extensão, vol. 6, no. 1, pp.19-27.

PEREIRA, E. M.; FARRAPEIRA, C. M. R.; PINTO, S. L. 2006. Percepção e educação ambiental sobre manguezais em escolas públicas da região metropolitana do Recife. Revista Eletrônica do Mestrado em Educação Ambiental, vol. 14, pp.17-15.

RODRIGUES, L. L.; FARRAPEIRA, C. M. R.; RODRIGUES, R. O. L. 2008. Percepção e educação ambiental sobre ecossistema manguezal incrementando as disciplinas de Ciências e Biologia em escola pública de Recife. Revista Investigações em Ensino de Ciências, vol. $13, \mathrm{n}^{\circ} .1$, pp.79-93.

SCHAEFFER-NOVELLI, Y.; CINTRÓN, G. 1986. Guia para estudo de áreas de manguezal: estrutura, função e flora. $2^{\mathrm{a}}$ ed., 431p., Editora Caribbean Ecological Research, São Paulo, SP, Brasil.

SCHEINER, T. C. M. 1984. O uso educativo da paisagem: educação ambiental e interpretação da natureza. Boletim da Fundação Brasileira para a Conservação da Natureza, vol. 19, pp.180-191.

SEDOVIM, W. R. M.; MAGALHÃES, L. M. F.; COSTA, O. G. 2005. Mergulhando na lama: uma experiência de aprendizagem, vivência e lazer no manguezal de Candeua-Curuçá/PA. In: VII Congresso de Ecologia do Brasil, Caxambu, MG, Brasil, p.806-808.

SEGALLA, M. B. 2008. "Legislinho e sua turma no manguezal" em sala de aula: Contribuições para a educação ambiental. Dissertação de Mestrado, Universidade do Vale do Itajaí, Itajaí, Brasil, 115p.

SILVA, M. M. M.; SOUSA, J. C. 2004. Ecoturismo e educação ambiental em áreas de manguezais na baía de Macapá, Luis Correia, Piauí. In: III Encontro de Pesquisa em Educação da UFPI, Teresina, PI, Brasil, pp.51-65.

SPALDING, M.; BLASCO, F.; FIELD, C. 1997. World Mangrove Atlas. ISME, Okinawa, Japan, 178p.

VIEIRA, B. P.; DIAS, D.; HANAZAKI, N. 2011a. Homogeneidade de encalhe de resíduos sólidos em um manguezal da Ilha de Santa Catarina, Brasil. Revista da Gestão Costeira Integrada, 11 (1): 21-30. 
VIEIRA, B. P.; DIAS, D.; NAKAMURA, E. M.; LEAL, M. L.; SILVA, T. G. R.; PIRES, C. D. O.; ARAI, T. I.; MINATELLI, S. Y. I.; CONCEIÇÃO, I. L.; HANAZAKI, N. 2011 b. Estudo quali-quantitativo de resíduos em manguezal de Florianópolis - SC. In: XIV Congresso Latino-Americano de Ciências do Mar, Balneário Camboriú, SC, Brasil, pp.1-3.

\section{AGRADECIMENTOS}

Walter Martin Widmer, Karla Z. Scherer, Carlos Danilo de Oliveira Pires, Indionara Lima Conceição, Lais da Silva Bellettini, Mariana Coutinho Hennemann, Mayana Lacerda Leal, Sabrina Yuri Imada Minatelli, Tammy Iwasa Arai e Thais Gabriella Reinert da Silva pelas diversas contribuições na realização do Projeto Manguezal. À Pró-Reitoria de Assuntos Estudantis (PRAE-UFSC) pela confecção do material gráfico para a realização das atividades de educação ambiental. E ao $\mathrm{CNPq}$ pela bolsa de produtividade em pesquisa à Natalia Hanazaki. 